\title{
The Improvement of Patient Centering in Computed Tomography Through a Technologist-Focused Education Initiative
}

\author{
Lindsay DeWeese $^{1}$ (D) $\cdot$ Thomas Griglock $^{1} \cdot$ Alexander Moody $^{1,2} \cdot$ Aaron Mehlberg $^{1,3} \cdot$ Celeste Winters $^{1}$
}

Received: 12 October 2021 / Revised: 19 November 2021 / Accepted: 28 December 2021 / Published online: 12 January 2022

(c) The Author(s) under exclusive licence to Society for Imaging Informatics in Medicine 2022

\begin{abstract}
Proper patient centering is fundamental to the operation of CT. Misalignment of the patient is known to have a negative impact on image quality and dose. The purpose of this study was to improve patient centering in CT and examine the efficacy of several educational methods that could be implemented at any clinical site. The IRB determined the study was not human subjects research, and oversight was waived. Three interventions were examined. The first intervention involved a discussion on patient centering at a staff meeting. As the second intervention, an educational presentation was developed and delivered to CT technologists addressing the physics behind the importance of patient centering in CT. As the third intervention, individual technologist centering performance reviews were conducted by the modality supervisor. Clinical scan data was collected for each study period via a cloud-based software to examine the efficacy of each intervention in terms of lateral and vertical offset. The mean vertical offset of the baseline data was $-1.97 \mathrm{~cm}$. After the staff meeting, the mean vertical offset decreased to $-1.60 \mathrm{~cm}(p<0.001)$. Following the educational presentation, the mean vertical offset decreased to $-1.14 \mathrm{~cm}$ $(p<0.001)$. After the technologist performance reviews, the mean vertical offset decreased to $-0.86 \mathrm{~cm}(p<0.001)$. This research examined a quality improvement initiative to improve patient centering at our institution which focused on communication and education. Through this initiative, the mean vertical positioning error decreased, the percentage of exams within $0-1 \mathrm{~cm}$ of isocenter increased, and the percentage of exams misaligned by greater than $3 \mathrm{~cm}$ decreased. This work has shown that patient centering can be improved with education.
\end{abstract}

Keywords Computed tomography · Patient centering · Dose monitoring software · Radiology education · Quality improvement

Lindsay DeWeese

sinclail@ohsu.edu

Thomas Griglock

griglock@ohsu.edu

Alexander Moody

moodya@livemail.uthscsa.edu

Aaron Mehlberg

aaron.d.mehlberg.mil@mail.mil

Celeste Winters

learyc@ohsu.edu

1 Department of Diagnostic Radiology, Oregon Health \& Science University, Portland, OR, USA

2 University of Texas Health Science Center at San Antonio, San Antonio, TX, USA

3 Naval Medical Center Portsmouth-Tricare Portsmouth, Portsmouth, VA, USA

\section{Introduction}

Accurate patient centering is essential to the proper clinical use of computed tomography (CT). Misalignment of the patient can result in deleterious effects on both the dose utilized to perform the CT scan and the resultant image quality of the scan.

Bowtie filtration is utilized in CT to decrease signal variation due to the difference in patient attenuation as the $\mathrm{x}$-ray tube rotates around the patient. Bowtie filters are thinnest in the center and gradually increase in thickness toward the periphery. The thinnest portion of the bowtie filter is designed to align with the thickest portion of the patient so as to maximize the intensity of the $\mathrm{x}$-ray beam in this anatomical area. Image quality issues have been observed when patients are not centered properly within the CT gantry due to improper alignment with bowtie filters. A study by Szczyktuowicz et al. found that when alignment is off by 
$10 \mathrm{~cm}$, noise in a single axial slice can vary by a factor of two, and CT numbers can vary by greater than $20 \mathrm{HU}$ [1]. Another study found a 10-16.5\% maximal increase in noise in abdominal CT images when patient misalignment was greater than $3 \mathrm{~cm} \mathrm{[2].}$

Noise and surface dose variation can be impacted by bowtie filter selection and patient size. Toth et al. showed a difference in noise variation for different-sized filters. A more substantial increase in noise was found for small bowtie filters for misalignment errors of 3 and $6 \mathrm{~cm}$ [3]. Clinically, this finding has image quality implications for small adult and pediatric patient populations. In a phantom-based study, surface dose measurements were found to increase by $21.9 \%$ on average for typical patient positioning errors in the vertical direction below isocenter [4].

Tube current modulation algorithms are utilized in the majority of adult body CT exams and are impacted by patient positioning errors. The radiation output needed for a scan is determined by the tube current modulation algorithm following the acquisition of the scan localizer. Patient size is estimated from the scan localizer. When a patient is not centered within the gantry, incorrect estimates of patient size can result and then impact the radiation output prescribed for the scan. One study found that misalignment in the vertical direction could result in size estimate errors for a phantom of up to a factor of 1.5 , which resulted in changes in the dose indices, Volume Computed Tomography Dose Index $\left(\mathrm{CTDI}_{\mathrm{Vol}}\right)$, and Size-Specific Dose Estimate (SSDE) of up to a factor of 4.4 and 2.7, respectively [5].

Multiple vendors have developed technology to improve patient-centering accuracy. One vendor employs a positioning compensation system (PCS) that automatically adjusts the tube current modulation algorithm when the scout image indicates a patient is larger or smaller than the actual patient size. A study by Barreto et al. observed the impact of the PCS on organ doses measured directly in post-mortem subjects; it was found that vertical misalignment of up to $4 \mathrm{~cm}$ resulted in a maximum organ dose difference of 35\% compared with the organ doses from a properly centered subject. When PCS was employed, the maximum difference in organ doses changed from 35 to $18 \%$. This finding indicates that although the use of the PCS reduced the impact of misalignment on organ dose variability, differences still exist for subjects that are not centered accurately [6].

In a recent advancement, one vendor utilizes a 3D camera to detect body contours via infrared light and fits the camera data to an avatar [7]. The patient's isocenter is then determined based on the avatar and the body region to be scanned by artificial intelligence (AI) algorithms, and the table height is adjusted accordingly. One study examined an AI-based automatic positioning tool for scanning COVID-19 patients and found a 9\% image noise reduction and a $16 \%$ radiation dose reduction with the use of the positioning tool in comparison with manual positioning by the technologists [8].

While there are a variety of technological advancements currently available to improve patient-centering errors, these are generally only offered on new CT scanners or updated software versions. Since nearly all clinical sites have CT units of various ages and technologies, methods seeking to improve patient centering at the technologist level remain paramount.

Over the last decade, dose monitoring information systems have become a ubiquitous tool for dose management in CT. Recently, these information systems have expanded to other areas of practice improvement. A study by Heilmaier et al. explored the effects of placing an extra computer next to the CT console which displayed a dose monitoring software in real-time. The technologists reviewed the dose information on the software following each exam and made comments regarding software notifications. The software gave notifications when a dose threshold was exceeded or when a patient was off-center by more than $1 \mathrm{~cm}$ in the vertical or lateral direction. After the implementation of the real-time dose monitoring approach, patient positioning errors decreased by almost $50 \%$ [9].

As mentioned, other studies have utilized dose monitoring software to examine patient centering improvement; however, to our knowledge, no other studies have utilized software to investigate the impact of a technologist-focused education initiative. This study utilized a commercially available dose monitoring cloud-based information system, Imalogix ${ }^{\mathrm{TM}}$, to evaluate the effectiveness of several technologist-based methods aimed at improving patient alignment in CT. Imalogix records the vertical offset and lateral offset (difference from isocenter) for each acquisition sent to the system. These values are utilized to report a percent accuracy and peer percentile ranking for the site.

The purpose of this study was to evaluate the efficacy of several methods aimed at the improvement of patient centering in CT with a focus on technologist education that can be implemented at any clinical site regardless of $\mathrm{CT}$ vendor or access to advanced technology or software.

\section{Methods}

Before performing this study, the study protocol was submitted to the Institutional Review Board (IRB). The IRB determined the study is not research involving human subjects, and therefore, IRB oversight was not required.

Three methods were utilized in progression to study their impact on technologists' patient-centering performance. The first method consisted of a brief discussion about patient centering at a CT staff meeting by the modality supervisor. The second method was an educational presentation given 
by a Qualified Medical Physicist (QMP) discussing the physics behind proper patient positioning and how improper alignment can impact both dose and image quality. The third method involved individual CT technologist performance reviews conducted by the modality supervisor with a focus on technologist-specific patient-centering performance. Centering performance was examined for a total of twenty-one technologists.

Clinical scan data was collected from Imalogix for all diagnostic CT scanners at our institution from September 2018 through April 2020. The information collected included the following: date of study, protocol, acquisition number, vertical offset, lateral offset, and $\mathrm{CTDI}_{\mathrm{Vol}}$.

Imalogix was utilized to examine the efficacy of each method. The software computes the vertical and lateral offset from scout images at the most central point of the patient along the $z$-axis. For vertical offset, a positive value indicates the patient was above isocenter while a negative value indicates the patient was below isocenter. For lateral offset, a positive value indicates the patient was right of isocenter while a negative value indicates the patient was left of isocenter. Determination of offset values is only done for exams allowing for SSDE calculation, thus excluding offset data for heads, necks, extremities, bolus scans, and exams that utilize fluoroscopy. Imalogix also displays percent accuracy and peer percentile ranking for each month. The percent accuracy is the percent of a facility's exams that were vertically centered within $0-3 \mathrm{~cm}$ of isocenter. For exams with multiple acquisitions, the offset for each acquisition is analyzed and if any of the acquisitions were within $0-3 \mathrm{~cm}$, the exam is considered accurate. Peer percentile ranking compares an institution's percent accuracy against other institutions that use Imalogix.

For this study, offset data was only counted once for each patient. If an exam consisted of multiple acquisitions, the offset data for the first scan was used. For combination exams consisting of a neck and chest protocol or neck and chest abdomen pelvis protocol, the information for the neck portion of the acquisition was not utilized as it did not contain offset data. In addition, all cardiac and spine scans were removed from the clinical scan dataset. The data was filtered by date to correspond with various time periods in the study as illustrated in Fig. 1.

Time period (TP) 1 was considered the baseline data and included scans from Jan 1, 2018 through Sept. 10, 2018. In a CT staff meeting conducted on September 10, 2018, the modality supervisor brought up the need to accurately center patients as an agenda item and reiterated the importance of double checking the position of the patient within the gantry prior to leaving the scan room. This brief discussion which lasted only a few minutes was defined as the first intervention to improve patient centering. TP 2 was the time between the CT staff meeting and the second intervention, which consisted of an educational presentation delivered to all CT technologists at our institution during the week of February 8, 2019. The educational presentation included information on current trends of lateral and vertical misalignment in CT, tips for proper patient alignment, and a review of the following key concepts: bowtie filtration, tube current modulation, and the impact of misalignment on dose and image quality (noise, CT number, and artifacts). The presentation was 60 min long. Data was evaluated 10 weeks after the presentation (TP 3) to determine the impact of the educational presentation on patient-centering accuracy.

During the week of January 27, 2020, one-on-one meetings were conducted between the CT supervisor and all CT technologists at our institution. A template was developed for these performance reviews which focused on technologistspecific patient-centering accuracy. The template consisted of a graphical display illustrating the percentage of exams in which each technologist achieved patient centering within $3 \mathrm{~cm}$ of isocenter, and the percentage of exams that was misaligned by $3-6 \mathrm{~cm}$ and greater than $6 \mathrm{~cm}$. Additionally, the template included data on centering accuracy on a protocol level, displaying the three best protocols and the three protocols where the greatest opportunity for improvement could be made. The last aspect of the performance reviews included a few specific cases where patients were misaligned by the greatest amount. These cases were reviewed with each technologist to discuss improvement opportunities. The performance review for each technologist was no longer than 30 min. Data was evaluated both 10 weeks before (TP 4)
Fig. 1 Timeline of each intervention along with corresponding time periods for data analysis

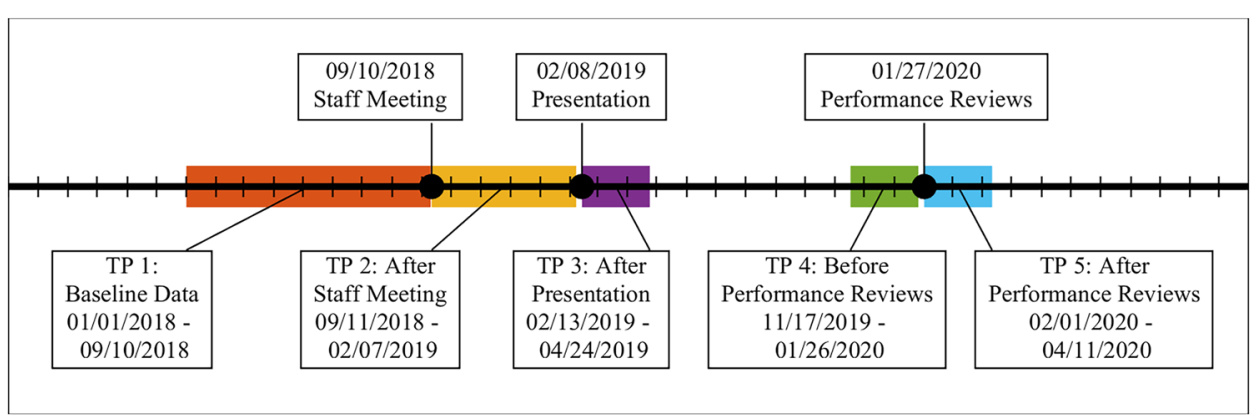


and 10 weeks following the CT technologist performance reviews (TP 5) to evaluate the effect on centering accuracy.

\section{Statistical Analysis}

A one-way Welch ANOVA was used to test for differences in means because the variance across groups was found to be inhomogeneous for all comparisons (Bartlett's test, $p<0.01$ ). Where significant differences were found, GamesHowell post hoc analysis was used to identify which groups had statistically significant difference in means. Results were considered statistically significant for $p$-values of 0.05 or lower.

\section{Results}

\section{Vertical Misalignment}

Table 1 summarizes the vertical misalignment data collected for each time period. The mean vertical offset was statistically significantly different between time periods (Welch's $F(4,9655.7)=190.08, p<0.001)$, and Games-Howell post hoc analysis revealed that all time periods had statistically significant differences in mean vertical offset.

Figure 2 displays a box plot of the vertical misalignment data in each group. The vertical misalignment improved after each intervention. The baseline data (TP 1) had a mean vertical misalignment of $-1.96 \mathrm{~cm}$ (SD 2.48). After the first intervention (staff meeting, TP2), the mean vertical misalignment of clinical exams was $-1.60 \mathrm{~cm}$ (SD 2.49), representing a change in mean vertical misalignment of $+0.37 \mathrm{~cm}$ (SE $0.26, p<0.001$ ). After the second intervention (educational presentation, TP3), the mean misalignment was $-1.14 \mathrm{~cm}$ (SD $2.32 \mathrm{~cm}$ ), indicating a change of $+0.46 \mathrm{~cm}$ (SE 0.035, $p<0.001(1.32 \mathrm{E}-11)$ ). Before the final intervention (TP 4 ), the mean vertical misalignment increased to -1.31 (SD $2.29)$, a change of $-0.17 \mathrm{~cm}$ (SE $0.039, p=0.02)$. After the technologist performance reviews (TP5), the mean vertical misalignment was $-0.86 \mathrm{~cm}$ (SD 2.3), indicating a change of $+0.45 \mathrm{~cm}(\mathrm{SE} 0.041, p<0.001)$.

Figure 3 depicts the percent of exams for each time period in the following vertical offset categories: $\leq 1 \mathrm{~cm},>1 \mathrm{~cm}-\leq$ $3 \mathrm{~cm},>3 \mathrm{~cm}-\leq 6 \mathrm{~cm}$, and $>6 \mathrm{~cm}$. As a result of the entire initiative, the percentage of exams aligned within $1 \mathrm{~cm}$ of isocenter increased from 23 to $34 \%$, while the percentage of exams misaligned by greater than $3 \mathrm{~cm}$ decreased from 36 to $20 \%$.

\section{Lateral Misalignment}

Table 2 summarizes the lateral misalignment data collected in the five time periods. Figure 4 displays a box plot of the lateral misalignment data. The difference in mean lateral

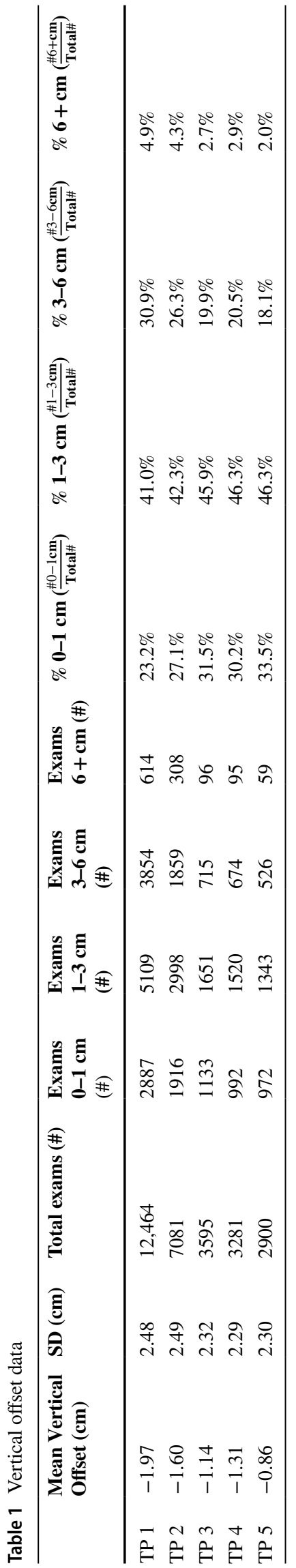




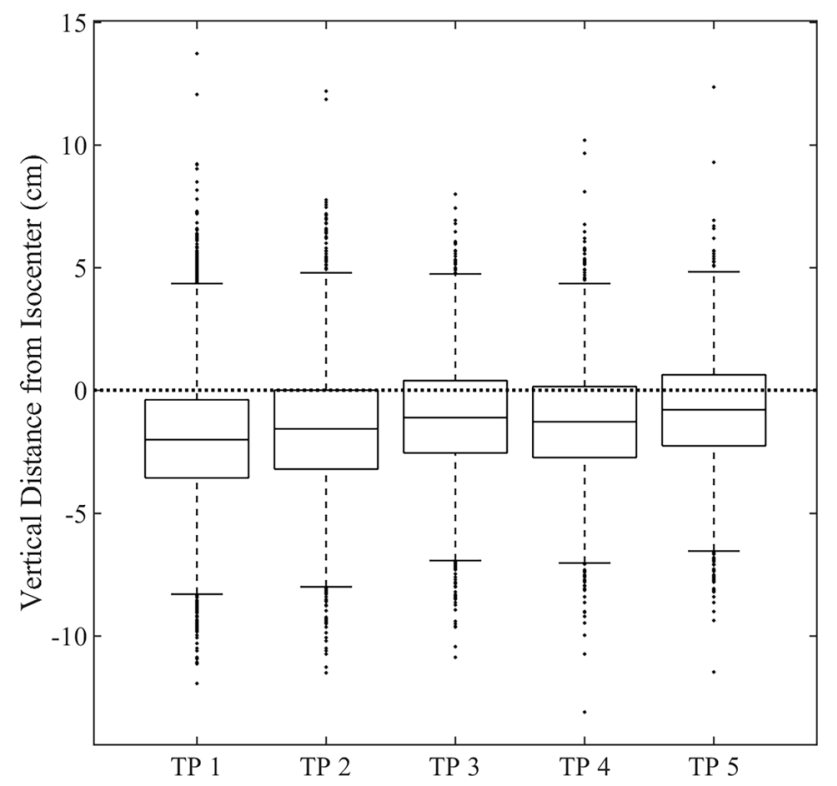

Fig. 2 Box plot of the vertical distance of the patient center from CT isocenter in the five studied time periods. The dotted line at $y=0$ indicates zero vertical offset. The median value is indicated with the central horizontal line through the boxes. The bottom and top of each box represent the 25 th and 75 th percentile of each distribution, respectively, and the whiskers represent 1.5 times the interquartile range. Outliers are depicted as dots beyond the whiskers. It is apparent from this plot that the vertical distance from isocenter was reduced after each intervention

offset between different time periods was statistically significant (Welch's $F(4,9576.2)=3.92, p=0.003)$. Games-Howell post hoc analysis revealed that the mean lateral misalignment in TP 1 (mean $=-0.18, \mathrm{SD}=1.52$ ) was statistically significantly different from the mean lateral misalignment in TP $2($ mean $=-0.12, \mathrm{SD}=1.51)$ and TP $3($ mean $=-0.09$, $\mathrm{SD}=1.43)$, representing changes of $0.06 \mathrm{~cm}(\mathrm{SE} 0.016 \mathrm{~cm}$, $p=0.03)$ and $0.09 \mathrm{~cm}(\mathrm{SE} 0.02, p=0.01)$, respectively. All other group comparisons were not statistically significant.

\section{Dose Index Analysis}

$\mathrm{CTDI}_{\mathrm{Vol}}$ data is summarized in Table 3, and histograms of $\mathrm{CTDI}_{\mathrm{Vol}}$ in each time period are displayed in Fig. 5. While the distributions of vertical and lateral misalignment were approximately normal, the $\mathrm{CTDI}_{\mathrm{Vol}}$ distributions were strongly right skewed (skewness 24-37). Due to this, CTDI $_{\mathrm{vol}}$ was logarithmically transformed to be approximately normal before analysis. Statistically significant differences between groups were indicated by Welch's ANOVA (Welch's $F(4,9491.4)=4.92$, $p<0.001)$. Games-Howell post hoc multiple comparison revealed that TP5 $\left(\log _{10}\left(\mathrm{CTDI}_{\mathrm{Vol}}\right)=1.06, \mathrm{SD}=0.24\right)$ had statistically significantly greater $\log _{10}\left(\mathrm{CTDI}_{\mathrm{Vol}}\right)$ than TP1 $\left(\log _{10}\left(\mathrm{CTDI}_{\mathrm{Vol}}\right)=1.05, \mathrm{SD}=0.24, p=0.006\right), \mathrm{TP} 2$ $\left(\log _{10}\left(\mathrm{CTDI}_{\mathrm{Vol}}\right)=1.04, \mathrm{SD}=0.25, p=0.005\right)$, and TP3 $\left(\log _{10}\left(\mathrm{CTDI}_{\mathrm{Vol}}\right)=1.04, \mathrm{SD}=0.26, p=0.02\right)$. This is reflected in the mode of $\mathrm{CTDI}_{\mathrm{Vol}}$ (peak of the distribution), which was $8.60 \mathrm{mGy}$ in TP5 and $3.70 \mathrm{mGy}$ in TP 1, TP 2, and TP 3.

Figure 6 shows the percent accuracy and peer percentile ranking for baseline and following each intervention. Imalogix displays this information on a monthly basis, and the months utilized are indicated on the figure. The percent accuracy increased from $61 \%$ at baseline to $80 \%$ at the end of the quality improvement initiative. This corresponds to an increase of 41 percentile points in terms of peer ranking.

\section{Discussion}

Accurate patient centering in CT ensures optimal performance of this advanced imaging modality. The optimization of image quality and dose are reliant on patient alignment within the CT scanner. Upon analysis of the baseline data which included 12,464 CT scans, the mean misalignment of clinical exams in the vertical direction was $-1.96 \mathrm{~cm}$ (SD $2.48 \mathrm{~cm}$ ). This is comparable to what other studies have
Fig. 3 Bar chart of percent of exams in each vertical misalignment category for each time period. The percent of exams with vertical offset $\leq$ $3 \mathrm{~cm}$ increased throughout the initiative, while the percent with misalignment $>3 \mathrm{~cm}$ decreased

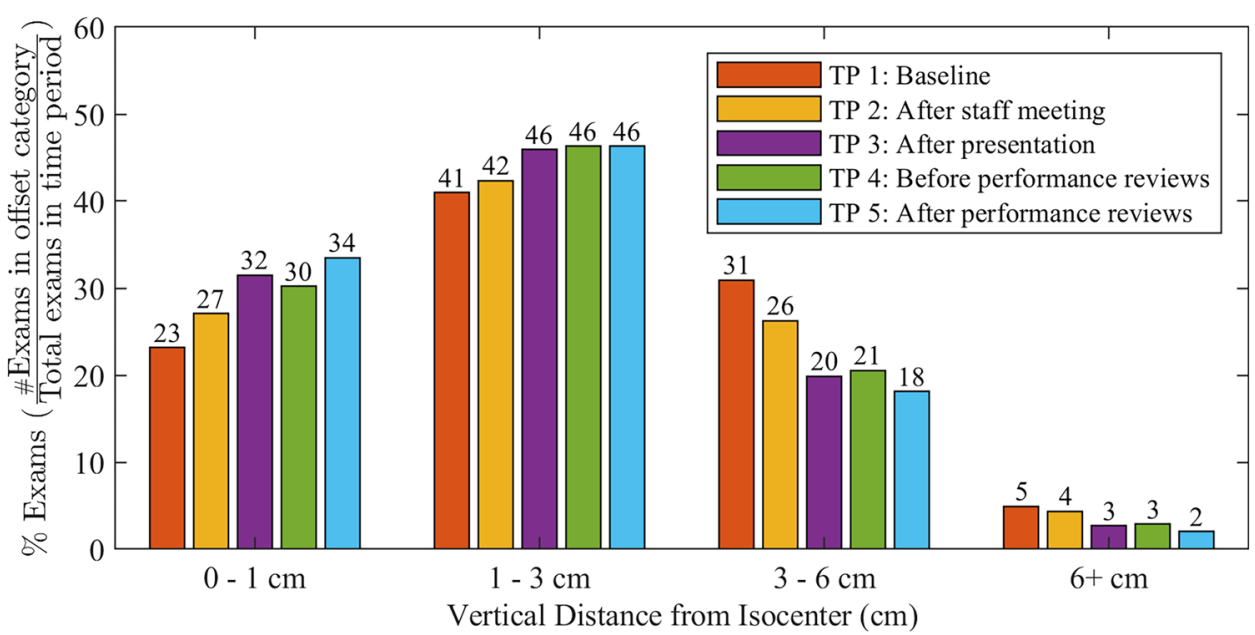



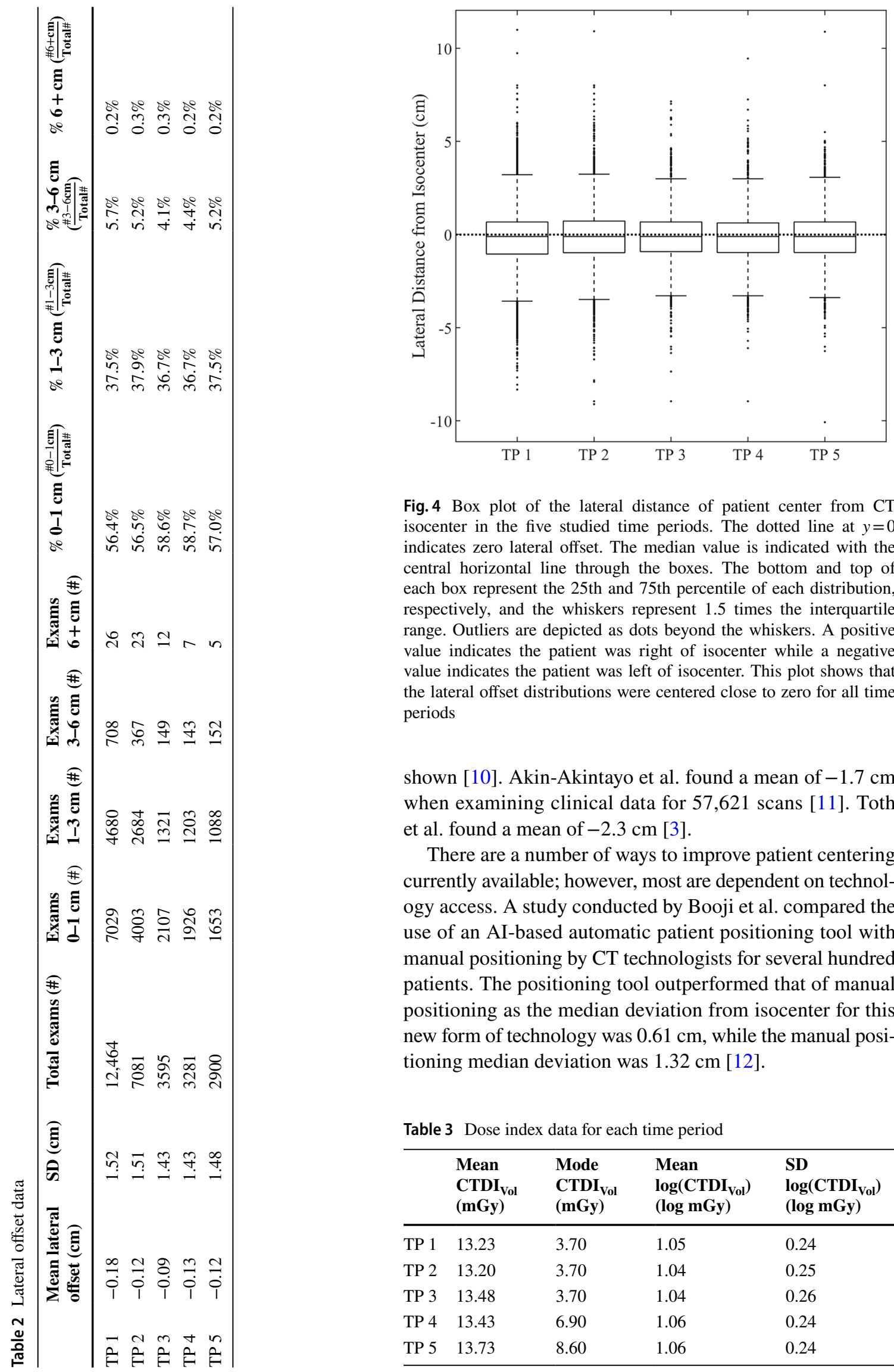

Fig. 4 Box plot of the lateral distance of patient center from CT isocenter in the five studied time periods. The dotted line at $y=0$ indicates zero lateral offset. The median value is indicated with the central horizontal line through the boxes. The bottom and top of each box represent the 25th and 75th percentile of each distribution, respectively, and the whiskers represent 1.5 times the interquartile range. Outliers are depicted as dots beyond the whiskers. A positive value indicates the patient was right of isocenter while a negative value indicates the patient was left of isocenter. This plot shows that the lateral offset distributions were centered close to zero for all time periods

shown [10]. Akin-Akintayo et al. found a mean of $-1.7 \mathrm{~cm}$ when examining clinical data for 57,621 scans [11]. Toth et al. found a mean of $-2.3 \mathrm{~cm} \mathrm{[3].}$

There are a number of ways to improve patient centering currently available; however, most are dependent on technology access. A study conducted by Booji et al. compared the use of an AI-based automatic patient positioning tool with manual positioning by CT technologists for several hundred patients. The positioning tool outperformed that of manual positioning as the median deviation from isocenter for this new form of technology was $0.61 \mathrm{~cm}$, while the manual positioning median deviation was $1.32 \mathrm{~cm} \mathrm{[12].}$

Table 3 Dose index data for each time period

\begin{tabular}{lllll}
\hline & $\begin{array}{l}\text { Mean } \\
\mathbf{C T D I}_{\mathbf{V o l}} \\
(\mathbf{m G y})\end{array}$ & $\begin{array}{l}\text { Mode } \\
\mathbf{C T D I}_{\mathbf{V o l}} \\
(\mathbf{m G y})\end{array}$ & $\begin{array}{l}\text { Mean } \\
\log (\mathbf{C T D I} \\
\left(\log \mathbf{~ m G y}_{\mathbf{V o l}}\right)\end{array}$ & $\begin{array}{l}\text { SD } \\
\log \left(\mathbf{C T D I}_{\mathbf{V o l}}\right) \\
(\log \mathbf{~ m G y})\end{array}$ \\
\hline TP 1 & 13.23 & 3.70 & 1.05 & 0.24 \\
TP 2 & 13.20 & 3.70 & 1.04 & 0.25 \\
TP 3 & 13.48 & 3.70 & 1.04 & 0.26 \\
TP 4 & 13.43 & 6.90 & 1.06 & 0.24 \\
TP 5 & 13.73 & 8.60 & 1.06 & 0.24 \\
\hline
\end{tabular}




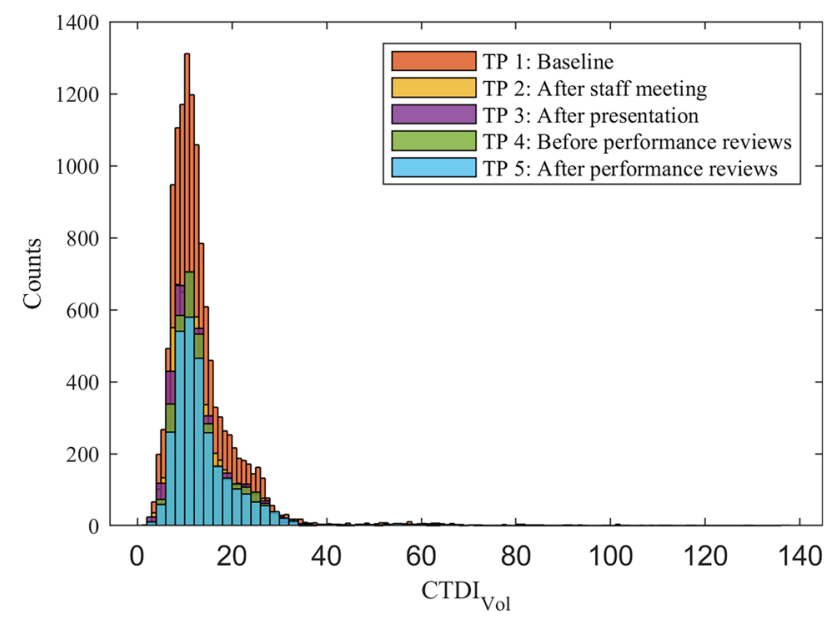

Fig. 5 Histograms of $\mathrm{CTDI}_{\mathrm{Vol}}$ in each time period studied display the strongly right-skewed distributions

The three methods presented with this work seek to improve patient-centering accuracy and have the ability to be implemented regardless of CT scanner technology or software currently available. When examining the data for all three methods of intervention, all methods were found to have a positive impact on the improvement of vertical patient misalignment in CT. Through the implementation of each method, the mean vertical misalignment decreased and the percentage of exams that were within $1 \mathrm{~cm}$ of isocenter and $3 \mathrm{~cm}$ of isocenter increased. In addition, the percentage of exams misaligned by greater than $6 \mathrm{~cm}$ decreased. This is a notable result as this degree of misalignment has been shown to impact both image quality and dose to the greatest extent [1,5].

It should also be noted that the effects of implementing this initiative were long term; there was a decrease in patientcentering improvement between the educational presentation and prior to the individual technologist performance reviews (mean vertical offset $-1.14 \mathrm{~cm}$ vs $-1.31 \mathrm{~cm}$ ). Still, the patient positioning errors were substantially improved from the baseline data demonstrating that consistent effort should be put into technologist education. Our institution plans to continue these efforts on an annual basis to prolong our improved patient-centering performance.

Technologist centering of patients in the lateral direction was consistently more accurate than centering in the vertical direction, as the mean lateral offset for the baseline data was $-0.18 \mathrm{~cm}$ (SD $1.52 \mathrm{~cm}$ ). Improvements in lateral misalignment were very minor for the entire initiative. The improvement was only statistically significant as a result of the first intervention which decreased the lateral offset to $-0.12 \mathrm{~cm}(p=0.03)$. Previous analysis of clinical scan data has also shown that lateral misalignment is minimal. One study found that the mean lateral offset was within $0.3 \mathrm{~cm}$ of isocenter [10], while another study found a mean lateral offset of $0.1 \mathrm{~cm} \mathrm{[3].}$

Upon analysis of the dose index data, no statistically significant change in dose index was observed for any of the interventions (TP1 to TP2, TP2 to TP3, and TP4 to TP5). A study by Cheng on a Philips (Philips Healthcare, Andover, MA, USA) Brilliance 64 CT found only a slight increase in $\mathrm{CTDI}_{\mathrm{Vol}}$ for typical patient misalignment values of $2.8 \mathrm{~cm}$ relative to accurately centered patients [13]. This validates our study finding as the majority of the scanners examined in this study are from the same vendor. Additionally, Marsh and Silosky showed that $\mathrm{CTDI}_{\mathrm{Vol}}$ changes for phantom measurements were very minimal for Philips CT scanners even at maximum and minimum vertical positions [5]. While the mean vertical misalignment decreased as a result of each intervention included in this study, the amount was small and did not result in a meaningful impact on radiation output.

There were some limitations to this study: first, an image quality analysis was not included in this study; therefore, no evidence was provided that image quality was improved as a result of increasing centering accuracy. However, there are a
Fig. 6 Percent accuracy and peer ranking percentile for baseline clinical data and after each intervention. An increase in patient centering accuracy and peer ranking was seen throughout the educational initiative

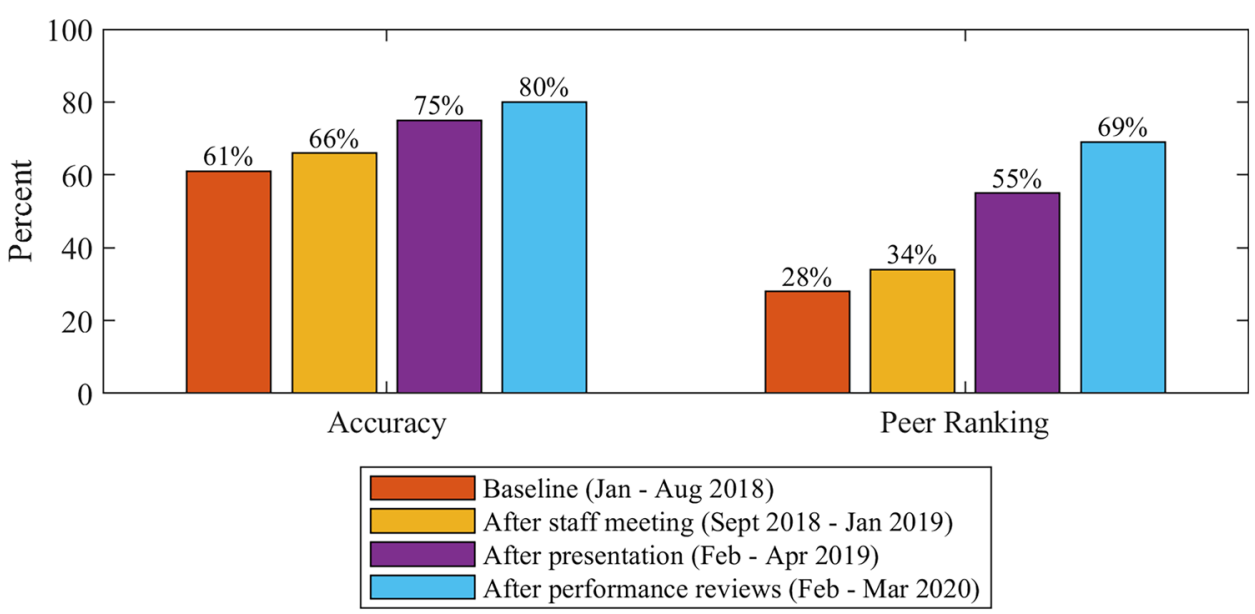


number of studies demonstrating a relationship between image quality and patient alignment $[1,4,10]$. Some institutions may not have access to dose monitoring software which tracks centering performance, inhibiting their availability to incorporate large amounts of data into a technologist performance review. In addition, the educational presentation is not standardized, and the efficacy of the methods could vary with the technologist sample size utilized. As such, the study methods may not be generalizable to other institutions. However, practice improvement could still be achieved through technologist education efforts and by performing a quality assurance check for all CT technologists and incorporating this information into an annual performance review. Additionally, this study only examined centering performance for $\mathrm{CT}$ scans where vertical and lateral offsets were estimated by Imalogix. As a result of the image analysis calculation method employed by the software, errors in the calculation of the offset are possible. Moreover, head CT scans were not able to be analyzed, which make up a large percentage of clinical scans and are likely to benefit from improved patient placement within the scanner. Lastly, while specific protocol performance was integrated into the technologist performance reviews, centering accuracy on a protocol level was not analyzed for the institution as a whole. The incorporation of this information could lead to further improvements in patient centering in CT.

\section{Conclusion}

This research examined several methods to improve patient centering at our institution which focused on enhancing communication and education opportunities with CT technologists. Through this quality improvement initiative, the mean vertical positioning error decreased, the percentage of exams within $0-1 \mathrm{~cm}$ of isocenter increased, and the percentage of exams misaligned by greater than $3 \mathrm{~cm}$ decreased. This work has shown that centering accuracy can be improved substantially with a technologist-focused approach.

Acknowledgements We would like to thank James Armbruster for providing information about Imalogix and insight into the software. The authors would also like to acknowledge our CT supervisor, Tyler Bogan, who provided significant support for this initiative.

\section{Declarations}

Ethics Approval The study protocol was submitted to the Institutional Review Board (IRB) at Oregon Health \& Science University. The IRB determined the study is not research involving human subjects and therefore IRB oversight was not required.
Conflict of Interest Thomas Griglock is a member of the Scientific Advisory Board for Imalogix ${ }^{\mathrm{TM}}$. No other authors have disclosures.

\section{References}

1. Szczykutowicz TP, DuPlissis A, Pickhardt PJ. Variation in CT Number and Image Noise Uniformity According to Patient Positioning in MDCT. AJR Am J Roentgenol. May 2017;208(5):10641072. https://doi.org/10.2214/AJR.16.17215

2. Kim MS, Singh S, Halpern E, Saini S, Kalra MK. Ablation margin assessment of liver tumors with intravenous contrastenhanced C-arm computed tomography. World J Radiol. Mar 28 2012;4(3):102-8. https://doi.org/10.4329/wjr.v4.i3.102

3. Toth T, Ge Z, Daly MP. The influence of patient centering on CT dose and image noise. Med Phys. Jul 2007;34(7):3093-101. https://doi.org/10.1118/1.2748113

4. Habibzadeh MA, Ay MR, Asl AR, Ghadiri H, Zaidi H. Impact of miscentering on patient dose and image noise in $\mathrm{x}$-ray CT imaging: phantom and clinical studies. Phys Med. Jul 2012;28(3):191-9. https://doi.org/10.1016/j.ejmp.2011.06.002

5. Marsh RM, Silosky MS. The effects of patient positioning when interpreting CT dose metrics: A phantom study. Med Phys. Apr 2017;44(4):1514-1524. https://doi.org/10.1002/mp.12137

6. Barreto I, Lamoureux R, Olguin C, et al. Impact of patient centering in $\mathrm{CT}$ on organ dose and the effect of using a positioning compensation system: Evidence from OSLD measurements in postmortem subjects. Journal of Applied Clinical Medical Physics. 2019;20(6):141-151. https://doi.org/10.1002/acm2.12594

7. Saltybaeva N, Schmidt B, Wimmer A, Flohr T, Alkadhi H. Precise and Automatic Patient Positioning in Computed Tomography. Investigative Radiology. 2018;53(11):641-646. https://doi.org/10.1097/rli. 0000000000000482

8. Gang Y, Chen X, Li H, et al. A comparison between manual and artificial intelligence-based automatic positioning in CT imaging for COVID-19 patients. European Radiology. 2021. https://doi. org/10.1007/s00330-020-07629-4

9. Heilmaier C, Zuber N, Bruijns B, Weishaupt D. Does Real-Time Monitoring of Patient Dose With Dose Management Software Increase CT Technologists' Radiation Awareness? AJR Am J Roentgenol. May 2016;206(5):1049-55. https://doi.org/10.2214/AJR.15.15466

10. Kaasalainen T, Palmu K, Reijonen V, Kortesniemi M. Effect of patient centering on patient dose and image noise in chest CT. AJR Am J Roentgenol. Jul 2014;203(1):123-30. https://doi.org/ 10.2214/AJR.13.12028

11. Akin-Akintayo OO, Alexander LF, Neill R, et al. Prevalence and Severity of Off-Centering During Diagnostic CT: Observations From 57,621 CT scans of the Chest, Abdomen, and/or Pelvis. Curr Probl Diagn Radiol. May - Jun 2019;48(3):229-234. https:// doi.org/10.1067/j.cpradiol.2018.02.007

12. Booij R, Budde RPJ, Dijkshoorn ML, Van Straten M. Accuracy of automated patient positioning in CT using a 3D camera for body contour detection. European Radiology. 2019;29(4):2079-2088. https://doi.org/10.1007/s00330-018-5745-z

13. Cheng PM. Patient Vertical Centering and Correlation with Radiation Output in Adult Abdominopelvic CT. J Digit Imaging. Aug 2016;29(4):428-37. https://doi.org/10.1007/s10278-016-9861-5

Publisher's Note Springer Nature remains neutral with regard to jurisdictional claims in published maps and institutional affiliations. 